\title{
PREVALENCE ET FACTEURS ASSOCIES A L'HALITOSE BUCCALE : ETUDE DANS UNE POPULATION GENERALE SENEGALAISE
}

\author{
B. FAYE, D. FAYE*, EB. MBODJ**, CMM. LO*, M. SARR, A.W KANE, \\ A.B DIAW***, BK. DIALLO**** \\ SERVICE D'ODONTOLOGIE CONSERVATRICE CHU DAKAR, \\ * SERVICE D'ODONTOLOGIE PRÉVENTIVE ET SOCIALE CHU DAKAR, \\ ** SERVICE DE PROTHĖSE DENTAIRE CHU DAKAR, \\ *** SERVICE D'ODONTOLOGIE HÔPITAL CAMBERENE DAKAR, \\ **** SERVICE D'ORL CHU DAKAR. SÉNÉGAL.
}

\begin{abstract}
Introduction: L'halitose ou mauvaise haleine est un trouble fréquent, chronique chez $50 \%$ de la population adulte, et d'origine buccale dans $90 \%$ des cas. L'objectif de cette étude était d'étudier la prévalence et les facteurs associés à l'halitose buccale dans une population générale sénégalaise.

Matériel et méthode : il s'agissait d'une étude descriptive transversale qui portait sur 396 patients sénégalais tous volontaires pour participer à l'étude. Nous avions utilisé une fiche de recueil de données comprenant 24 questions sur l'autoévaluation de la mauvaise haleine. Ensuite tous les sujets étaient examinés pour évaluer les moyennes organoleptiques et la mesure des composés volatils sulfurés, grâce à l'halimétre. L'halitose était diagnostiquée dés que le niveau de Composés Volatiles Sulfurés était $\geq$ à $125 \mathrm{ppb}$ et le score organoleptique $\geq 2$. L'analyse statistique était réalisée en utilisant le test du chi2.

Résultats : La prévalence globale de l'halitose était de 32,3\%. La différence n'était pas statistiquement significative entre l'homme et la femme. Sur les 396 patients présentant une mauvaise haleine,128 avaient une halitose permanente ; 52 souffraient d'une halitose physiologique. La moyenne des scores organoleptiques était de 3,12 et celle des Composés Volatils Sulfurés de 273,9 ppb.

Conclusion : Les résultats de l'étude avaient montré une corrélation entre les tests organoleptiques et le niveau de composés volatils sulfurés.
\end{abstract}

Mots clés : Halitose, tests organoleptiques, composés volatils sulfurés, épidémiology, autoévaluation.

\begin{abstract}
Introduction: Halitosis is a common complain among dental patients, chronic among $50 \%$ of the adult population, and of oral origin in $90 \%$ of the cases. The objective of this study was to evaluate the prevalence and related factors of oral halitosis among a senegalese population.

Material and method: This was a transversal descriptive study of 396 Senegalese adults using a 24-point self-assessment questionnaire on halitosis. All subjects were examined to estimate the organoleptic means and to measure the Sulfur Volatile Compounds (SVC), using a halimeter. Halitosis was diagnosed when the level of sulfur volatile Compound was $\geq$ $125 \mathrm{ppb}$ and the organoleptic score $\geq 2$. Data were statistically analyzed using the chi2 test.

Results: The global prevalence of halitosis was $32.3 \%$. The difference was not statistically significant between male and female. Among the 396 patients suffering from bad breath, 128 had a permanent halitosis and 52 had a physiological halitosis. The means of the organoleptic scores were 3.12 and that of the (SVC) were $273.9 \mathrm{ppb}$.

Conclusion: The results of the study showed a relation between the organoleptic tests and the level of sulfur volatile compounds (SVC).

Keywords: Halitosis, organoleptic tests, sulfur volatile compound, epidemiology, and self-assessment.
\end{abstract}

\section{INTRODUCTION}

L'halitose ou mauvaise haleine est un problème qui traverse I'histoire, la culture, la race et le sexe (1). Du point de vue épidémiologique, elle touche $50 \%$ de la population adulte. II s'agit d'une situation connue et évoquée depuis les époques romaine et grecque (1). Ainsi, Hippocrate (344-460 av. J.-C.) avait déjà décrit des recettes de bains de bouche pour lutter contre la mauvaise haleine. C'est un trouble rencontré dans le monde entier et lié dans $90 \%$ des cas à une surcharge bactérienne dans la cavité buccale. Les autres causes relèvent de l'Oto-RhinoLaryngologie et de maladies respiratoires. Les affections gastro-intestinales ou rénales et les syndromes métaboliques sont des causes mineures (2). Selon les facteurs étiologiques qui la provoquent, l'halitose peut être physiologique ou pathologique. L'halitose physiologique est due 
à la flore bactérienne peuplant la cavité buccale et n'est que temporaire lorsque des mesures d'hygiène adéquates sont mises en place. L'halitose pathologique est quant à elle permanente et ne peut être traitée que par une approche thérapeutique adéquate (3). Elles entraînent toutes les deux chez la personne qui en souffre un handicap social et psychologique. Cependant ce n'est que seulement au cours de ces dernières années, quand le problème a fortement touché l'intérêt public que la communauté scientifique a commencé à s'y intéresser de plus prés (3). Des investigations épidémiologiques précises sont en cours. Les observations proviennent soit des constatations que les médecins ou les chirurgiens-dentistes ont faites auprès de leurs patients, soit d'enquêtes d'organisations qui ont déterminé la fréquence de la mauvaise haleine pour un groupe donné $(4,5,6,7)$.

Le quart de la population occidentale souffre de mauvaise haleine tenace (7).

En Afrique, les études la concernant sont rares et elle y reste encore un sujet tabou.

Au Sénégal, il n'existe actuellement aucune étude sur ce trouble.

Ce travail nous permettra de disposer de données sur la prévalence et les facteurs associés à l'halitose buccale sur une population générale Sénégalaise.

\section{MATÉRIEL ET MÉTHODES}

II s'agissait d'une étude descriptive et transversale qui concernait 396 sénégalais volontaires. L'étude s'était déroulée sur 5 mois ( Novembre 2008 - Mars 2009). Elle avait porté sur tous les patients venus en consultation au service d'Odontologie Conservatrice du Département d'Odontologie de la Faculté de Médecine de Dakar et aux districts sanitaires de Hann, Cambérene et Gaspard Camara. Les sujets étaient auparavant informés sur la nature de l'étude et avaient signé un consentement éclairé approuvé par la commission nationale d'éthique du Ministère de la Santé et de la Prévention médicale du Sénégal.

Les personnes recrutées devaient ensuite remplir un questionnaire structuré en 24 items et dont le but était de recueillir toutes les données relatives à l'anamnèse médicale, à l'anamnèse dentaire et à l'halitose. Ce questionnaire devait permettre d'exclure les causes psychosomatiques de la mauvaise haleine. Les questions suivantes ont été étudiées :

- Les antécédents médicaux connus en rapport avec les maladies infectieuses, gastriques, une éventuelle opération chirurgicale, les allergies ou la prise de médicaments. - Les pratiques d'hygiène bucco-dentaire : Combien de fois par jour vous vous brossez les dents ? Avez utilisez la soie dentaire (oui / non)? Nettoyez-vous votre langue (oui / non)? Utilisez-vous un bain de bouche (oui / non)? - Combien de cigarettes fumez-vous par jour?

- Quelle est selon vous l'intensité de votre mauvaise haleine? (très forte, moyenne, faible)

- Comment savez-vous que vous avez une mauvaise haleine? (Langage du corps, d'autres personnes, je le sais tout simplement)

- Quel est selon vous votre état de stress ? (très élevé, élevé, moyen, faible)

- La mauvaise haleine a-t-elle une influence sur votre vie sociale ? (oui/non)

- Constatez-vous des dépôts sur votre langue? (oui/non)

- D'après vous, votre mauvaise haleine, vient-elle : de la bouche, du nez ou des deux?

- Comment traitez-vous votre mauvaise haleine? Aucun, dentifrice, nettoyage des espaces inter dentaires, gargarismes, dentiste, médecin ORL.

Par la suite, tous les patients étaient examinés pour avoir des données en vue de calculer les moyennes organoleptiques en utilisant l'échelle nominale en 5 paliers de Rosenberg (8). L'examen organoleptique était réalisé alors que le patient procédait à une inspiration et expiration non forcées. Le nez de l'examinateur était situé à une distance de $15 \mathrm{~cm}$ de celui du patient pour évaluer les odeurs de la bouche, du nez et des poumons.

- 0 Absence d'halitose

- 1 Mauvaise haleine légère

- 2 Mauvaise haleine modérée

- 3 Mauvaise haleine intense

- 4 Haleine extrêmement fétide

Par rapport à l'odeur buccale, nous avions demandé au patient de fermer la bouche et de respirer par le nez pendant $3 \mathrm{mn}$ afin que le maximum de gaz malodorants s'accumule dans la cavité buccale. Le sujet était alors invité à respirer doucement à une distance de $15 \mathrm{~cm}$ de l'interrogateur qui enregistre les scores organoleptiques.

Pour l'odeur du nez, l'évaluation était obtenue en demandant au patient d'inhaler par sa bouche et d'exhaler doucement par un nez puis par l'autre. Pour chaque exhalation, les scores organoleptiques avaient été déterminés.

Quant à l'odeur des poumons, il était demandé au patient de respirer par son nez en maintenant la bouche hermétiquement fermée pendant $3 \mathrm{mn}$. Après, le sujet était invité à exhaler, vidant autant que possible l'air des poumons, à une distance de $15 \mathrm{~cm}$ de l'examinateur.

Les niveaux de Composés Volatiles Sulfurés étaient quantifiés, une fois les évaluations organoleptiques complétées, à l'aide d'un moniteur portatif de composés sulfurés (Halimeter ${ }^{\circledR}$, Interscan Co., Chatsworth, USA). Le sujet était invité à fermer la bouche et à respirer par le nez pendant 3 minutes. L'examinateur tenait le brin de paille à une distance bien définie, selon le manuel d'instruction du fabricant. La position du capteur de I'halimétre avait permis en outre de détecter jusqu'à un certain point d'où provenait la mauvaise haleine (cavité buccale, langue, poumons ou nez). Le patient ne devait pas respirer lors de la lecture de Halimétre. Ce procédé était répété 3 fois et une moyenne des 3 lectures avait été calculée. La mauvaise 
haleine était diagnostiquée dés que la valeur était $\geq 125$ ppb (recommandations du fabricant) et le score organoleptique $\geq 2$. Nous avions utilisé la classification de Yaegaki et Coil (9).

Les données étaient ensuite saisies et analysées avec le logiciel Microsoft Access. L'analyse statistique était effectuée avec le logiciel S.P.S.S version 10.5.

Les comparaisons étaient faites avec le test du Chi2 avec un seuil de significativité $p<0,05$

\section{RESULTATS}

La moyenne d'âge de tous les participants était de $35,2 \pm 12.5$ années (avec un intervalle de confiance compris entre 20 à 70 ans). Les patients étaient répartis en 208 hommes (52,5\%) et 188 femmes $(47,5 \%)$.

La prévalence globale de l'halitose était de $32,3 \%$. En ce qui concerne le sexe, il n'y a pas de différence statistiquement significative $\mathrm{P}>0,05$.

La majorité des sujets présentant une halitose avait conscience d'avoir une très forte mauvaise haleine à travers leur propre perception $39,1 \%$ contre $26,1 \%$ d'halitose moyenne et $34,8 \%$ d'halitose faible.

Le niveau de stress était moyennement élevé chez 160 sujets soit $40,4 \%$.

Sur l'ensemble des patients interrogés, 61 n'avaient jamais fait de traitement de la mauvaise haleine (Tableau I). Pour les facteurs étiologiques associés à l'halitose, 104 incriminaient les causes d'origine buccale (tableau II) et 24 les dépôts au niveau de la langue (tableau II).

Pour l'halitose pathologique, le niveau moyen de Composés Volatils Sulfurés était $\geq 125 \mathrm{ppb}$ et les scores organoleptiques $\geq 2$. Pour l'halitose physiologique, le niveau moyen des Composés Volatils Sulfurés était $\geq 75$ ppb et les scores organoleptiques $\geq 1$. Sans halitose, le niveau moyen des Composés Volatils Sulfurés était $\leq 75$ $\mathrm{ppb}$ et les scores organoleptiques $=0$ ( tableau III).

\begin{tabular}{|c|c|c|}
\hline Questions & $\mathrm{N}$ & (\%) \\
\hline \multicolumn{3}{|c|}{ Savez vous que vous avez la mauvaise haleine? } \\
\hline Oui & 128 & $(32,3)$ \\
\hline Non & 268 & $(67,7)$ \\
\hline \multicolumn{3}{|c|}{ Quelle est selon vous fintensité de votre mauvalse haleine? } \\
\hline Très forte & 50 & $(39,1)$ \\
\hline Moyenne & 33 & $(26,1)$ \\
\hline Faible & 45 & $(34,8)$ \\
\hline \multicolumn{3}{|c|}{ Quel est selon vous votre sétat de stresss? } \\
\hline Tres eleve & 20 & $(5,1)$ \\
\hline Eleve & 76 & $(19,2)$ \\
\hline Moyen & 160 & $(40,4)$ \\
\hline Faible & 140 & $(35,4)$ \\
\hline \multicolumn{3}{|c|}{ La mauvaise haleine, a-t-elle une influence sur votre vie sociale? } \\
\hline Oui & 41 & $(31,8)$ \\
\hline Non & 87 & $(68,1)$ \\
\hline \multicolumn{3}{|c|}{ Avez-vous recu un traitement avant? } \\
\hline Oui & 67 & $(52,5)$ \\
\hline Non & 61 & $(47,5)$ \\
\hline
\end{tabular}

Tableau I: Halitose par auto-évaluation

\begin{tabular}{|l|c|c|}
\hline Facteurs & Nombre & Pourcentage \\
\hline Mauvaise haleine & 104 & 26 \\
Bouche & 16 & 8,9 \\
Nez & 60 & 33,3 \\
Bouche et nez & 88 & 22,2 \\
\hline Dépôt sur langue & 76 & 19,2 \\
\hline Sécheresse buccale & 72 & 18,2 \\
\hline Consommation de médicaments & & \\
\hline Tabagisme & 28 & 7,1 \\
Oui & 368 & 92,9 \\
Non & & \\
\hline Maladies gastriques & 128 & 32,3 \\
Oui & 268 & 67,7 \\
Non & & \\
\hline Sinusite chronique & 176 & 44,4 \\
Oui & 220 & 55,6 \\
Non & & \\
\hline
\end{tabular}

Tableau II: Facteurs étiologiques associés

\begin{tabular}{|c|c|c|c|}
\hline \multicolumn{2}{|c|}{ Mesure des CVS } & \multicolumn{2}{|c|}{ Evaluation des scores organoleptiques } \\
\hline $\mathrm{Ppb}$ & Sujets & Scores & Sujets \\
\hline$\leq 75$ & 194 & 0 & 216 \\
\hline 75- 125 & 74 & 1 & 52 \\
\hline $126-160$ & 36 & 2 & 42 \\
\hline$\geq 160$ & 92 & 3 & 48 \\
\hline & & 4 & 38 \\
\hline
\end{tabular}

Tableau III: Composés Volatils Sulfurés (CVS) et Scores organoleptiques de I'halitose selon les Indices de Rosenberg

\section{DISCUSSION}

Cette étude que nous avons menée a été la première réalisée dans une population sénégalaise. L'objectif était d'évaluer les facteurs associés à l'halitose par l'auto-évaluation et d'examiner son association avec des facteurs sociodémographiques, systémiques, et d'hygiène buccodentaire. Ces résultats étaient comparés aux tests organoleptiques et à la mesure des composés volatiles sulfurés par l'halimétre.

La prévalence de la mauvaise haleine dans cet échantillon de patients était de $32,3 \%$. Ce chiffre est comparable aux résultats rapportés dans d'autres populations, y compris les études effectuées en France, au Japon, et les estimations de la population générale des Etats-Unis $(10,11,12)$.

En effet, plusieurs auteurs rapportaient que $50 \%$ de la population nord-américaine souffrait de mauvaise haleine $(11,12)$. Une enquête téléphonique effectuée, afin de déterminer combien de personnes prenaient occasionnellement ou régulièrement des produits contre l'halitose (11) avait montré que $60 \%$ des femmes et $50 \%$ des hommes utilisaient des produits pour se rafraîchir l'haleine. $27,5 \%$ des personnes examinées en Chine se plaignaient de mauvaise haleine (13). Au Japon, parmi 232 répondants à une étude sur l'halitose, environ $47 \%$ 
étaient convaincus qu'ils avaient une mauvaise haleine (11).

Frexinos (10) avait rapporté en France un taux de prévalence de $22 \%$ de personnes qui souffraient de mauvaise haleine. Une étude de Seemann (14) en Allemagne sur 520 personnes trouve que : $76 \%$ souffraient occasionnellement de mauvaise haleine, $7 \%$ d'une mauvaise haleine permanente et $58 \%$ connaissaient des collègues qui souffraient de mauvaise haleine.

Parmi les antécédents médicaux inclus dans cette étude, les troubles gastro-intestinaux (100\%) et les sinusites chroniques $(44,4 \%)$ ont été significativement associés par auto-évaluation à la mauvaise haleine.

Il existe de nombreuses idées préconçues concernant l'étiologie de l'halitose (15). Les patients, mais aussi les praticiens, pensent à tort que la mauvaise haleine était d'abord due à des processus pathologiques au niveau du tractus gastro-intestinal. Ceci a été retrouvé dans notre étude où tous les sujets souffrant d'halitose permanente, avaient incriminé la maladie gastrique comme facteur étiologique. L'une des conséquences de cette conception erronée était le fait que les patients consultaient d'abord un gastro-entérologue, au lieu de médecin dentiste. Les origines gastro-intestinales de l'halitose sont plutôt rares; en effet, elles ne représentent qu'environ $1 \%$ de toutes les étiologies de cette affection (16). Les troubles gastrointestinaux associés comme causes extra-orales de la mauvaise haleine, peuvent être potentiellement dus à Helicobacter pylori (16). Cependant le rôle causal d'Helicobacter pylori dans le contexte de l'halitose n'a pas encore été établi avec certitude (18). Certains auteurs $(15,17,18)$ avaient pu observer une corrélation entre la présence d'Helicobacter pylori et l'halitose, d'une part, une observation étayée, d'autre part, par l'effet inverse d'une antibiothérapie ciblée contre Helicobacter pylori (17). Les effets d'une telle médication sur d'autres germes n'ont cependant pas été étudiés jusqu'à présent. Ce d'autant que chez les patients traités par des antibiotiques en raison d'une infection par Helicobacter pylori, aucune amélioration de l'halitose n'a été observée (18).

La sinusite chronique a également été rapportée par certaines études comme un facteur étiologique possible de mauvaise haleine (17). Dans notre étude environ $45 \%$ des patients l'ont incriminée. Pour Krespi (17) les affections de la sphère ORL représentent environ 5 à $8 \%$ de tous les facteurs étiologiques de l'halitose.

L'amygdalite chronique et la sinusite chronique sont les causes non buccales les plus fréquentes (19).

Dans de nombreux cas, les patients affectés rapportent qu'ils ressentent un écoulement postérieur permanent de sécrétions qui, dans le contexte de l'halitose, est incriminé en tant que facteur contribuant à la formation d'enduits sur la face dorsale de la langue $(20,21)$.

\section{CONCLUSION}

L'halitose est un fléau mondial qui touche tous les continents et toutes les tranches d'âge. Les hommes en ont souffert depuis l'antiquité. Cependant, elle n'a commencé à intéresser la communauté scientifique que récemment quand l'intérêt du public était menacé. Nos résultats fournissent des renseignements de base sur les plaintes de mauvaise haleine et indiquent l'ampleur de ce problème chez les sénégalais.

\section{REFERENCES}

1. Rosenberg M : Bad breath: Research perspectives. 2nd Edition, 1997. 2. Annemiek M.W.T, Van den Broek, Louw Feenstra, Cees de Baat A review of the current literature on aetiology and measurement methods of halitosis Journal of dentistry 2007; 35: 627-635

3. Rosenberg $M$ : Clinical assessment of bad breath: current concepts JADA 1996: 127: 475-82.

4. Miyazaki H, Sakao S, Katoh Y, Takehara T : Correlation between volatile sulphur compounds and certain oral health measurements in the general population. J. Periodontol 1995; 66: 679-84.

5. Nadanovsky P, Carvalho LBM Oral malodour and its association with age and sex in a general population in Brazil Oral Diseases 2007; 13: 105-9.

6. Loesche WJ, Kazor C. Microbiology and treatment of halitosis. Periodontol 2002; 28: 256-79.

7. Bornstein MM, Kislig K, Hoti BB, Seemann R, Lussi A. Prevalence of halitosis in the population of the city of Bern, Switzerland: A study comparing self-reported and clinical data. Eur J Oral Sci 2009; 117: 261-7.

8. Rosenberg M, Kulkarni G.V, Bosy A, McCulloch C.O.G Reproductibility and sensitivity of oral maladour measurements with a portable monitor sulphide monitor. J Dent Res1991; 70: 1436-40.

9. Yaegaki K, Coil JM Examination, classification, and treatment of halitosis; clinical perspectives. J Can Dent Assoc 2000; 66: 257-61.

10. Frexinos J., Denis P, Allemand H, Allouche $S$ : Descriptive study of digestive functional symptoms in the French general population. Gastroenterologie Clinique et biologique 1998; 22:785-91.

11. Morita M, Wang H.LAssociation between oral malodor and adult periodonti- tis : J Clin Periodontol 2001; 28: 813-819

12. Greenman J, El-Maaytah M :Assessing the relationship between concentrations of malodour compounds and odor scores from judges. JADA 2004; 136: 749-757. 13. Liu X, N Shinada K, Chen X C :Oral malodor-related parameters in the Chinese general population J Clin Periodontol 2006; 33: 31-36

14. Seemann R, Bizhang M, Djamchidi C, Nachnami S, Kage A.

High proportion of pseudohalitosis patients in a multidisciplinary breath malodor consultation. Int Dent J 2006; 56: 77-81.

15. Crohn BB, Drosd R. Origin of mouth odors - halitosis. New York J Dent 1942; 12: $192-197$.

16. Hoshi K, Yamano Y, Mitsunaga A, Shimizu S, Kagawa J.

Gastrointestinal diseases and halitosis: association of gastric Helicobacter pylori infection. International Dental Journal 2002; 52(Suppl. 3):207-11.

17. Krespi Y P, Shrime M.G, Kacker A.

The relationship between oral malodor and volatile sulfur compound-producing bacteria. Otolaryngology Head and Neck Surgery 2006; 135: 671-676.

18. Norfleet RG Helicobacter halitosis. J Clin Gastroenterol 1993; 16: 274. 19. Tangerman A, Winkel E .G Intra- and extra-oral halitosis: finding of a new form of extra-oral blood-borne halitosis caused by dimethyl sulphide $\mathrm{J}$ Clin Periodontol 2007; 34: 748-755

20. Christensen G.J Why clean your tongue?JADA 1998; 129: 1605-1607 21. Van den Broek M.W.T, Louw Feenstra, Cees de BaatOral malodor - a review Archives of oral biology 2008; 53 (1): 1-7 